\title{
A Mixed Line Search Smoothing Quasi-Newton Method for Solving Linear Second-Order Cone Programming Problem
}

\author{
Zhuqing Gui, ${ }^{1}$ Chunyan $\mathrm{Hu}^{2}$ and Zhibin $\mathrm{Zhu}^{1}$ \\ ${ }^{1}$ School of Mathematics and Computing Science, Guilin University of Electronic Technology, Guilin 541004, China \\ ${ }^{2}$ School of Electronic Engineering and Automation, Guilin University of Electronic Technology, Guilin 541004, China \\ Correspondence should be addressed to Chunyan Hu; huchyel@hotmail.com
}

Received 30 January 2013; Accepted 19 February 2013

Academic Editors: W. Bein, A. Piunovskiy, and G. Silva

Copyright (c) 2013 Zhuqing Gui et al. This is an open access article distributed under the Creative Commons Attribution License, which permits unrestricted use, distribution, and reproduction in any medium, provided the original work is properly cited.

Firstly, we give the Karush-Kuhn-Tucker (KKT) optimality condition of primal problem and introduce Jordan algebra simply. On the basis of Jordan algebra, we extend smoothing Fischer-Burmeister (F-B) function to Jordan algebra and make the complementarity condition smoothing. So the first-order optimization condition can be reformed to a nonlinear system. Secondly, we use the mixed line search quasi-Newton method to solve this nonlinear system. Finally, we prove the globally and locally superlinear convergence of the algorithm.

\section{Introduction}

Linear second-order cone programming (SOCP) problems are convex optimization problems which minimize a linear function over the intersection of an affine linear manifold with the Cartesian product of second-order cones. Linear programming (LP), Linear second-order cone programming (SOCP), and semidefinite programming (SDP) all belong to symmetric cone analysis. LP is a special example of SOCP and SOCP is a special case of SDP. SOCP can be solved by the corresponding to the algorithm of SDP, and SOCP also has effectual solving method. Nesterov and Todd $[1,2]$ had an earlier research on primal-dual interior point method. In the rescent, it gives quick development about the solving method for SOCP. Many scholars concentrate on SOCP.

The primal and dual standard forms of the linear SOCP are given by

$$
\begin{aligned}
& \text { Primal Dual } \\
& \min c^{T} x \quad \max b^{T} y \\
& \text { s.t. } A x=b, \quad \text { s.t. } A^{T} y+z=c \text {, } \\
& x \succcurlyeq_{K} 0, \quad z \succcurlyeq_{K} 0 \text {, }
\end{aligned}
$$

where the second-order cone $K$ :

$$
K=\left\{\left(x_{0} ; \bar{x}\right) \in R \times R^{n-1} \mid x_{0} \geq\|\bar{x}\|\right\}
$$

where $\|\cdot\|$ refers to the standard Euclidean norm.

In this paper, the vectors $x, c$, and $z$ and the matrix $A$ are partitioned conformally, namely

$$
\begin{gathered}
x=\left(x_{1} ; \ldots ; x_{r}\right) x_{i} \in R^{n_{i}}, \quad c=\left(c_{1} ; \ldots ; c_{r}\right) c_{i} \in R^{n_{i}}, \\
z=\left(z_{1} ; \cdots ; z_{r}\right) z_{i} \in R^{n_{i}}, \quad A=\left(A_{1} ; \cdots ; A_{r}\right) A_{i} \in R^{m \times n_{i}} .
\end{gathered}
$$

Except for interior point method, semismoothing and smoothing Newton method can also be used to solve SOCP. In [3], the Karush-Kuhn-Tucker (KKT) optimality condition of primal-dual problem was reformulated to a semismoothing nonlinear system, which was solved by Newton method with central path. In [4], the KKT optimality condition of primal-dual problem was reformed to a smoothing nonlinear equations, then it was solved by combining Newton method with central path. References $[3,4]$ gave globally and locally quadratic convergent of the algorithm. 


\section{Preliminaries and Algorithm}

In this section, we introduce the Jordan algebra and give the nonlinear system, which comes from the Karush-KuhnTucker (KKT) optimality condition. At last, we introduce two kinds of derivative-free line search rules.

Associated with each vector $x \in R^{n}$, there is an arrowshaped matrix $\operatorname{Arw}(x)$ which is defined as follows:

$$
\operatorname{Arw}(x)=\left(\begin{array}{cc}
x_{0} & \bar{x}^{T} \\
\bar{x} & x_{0} I
\end{array}\right) .
$$

Euclidean Jordan algebra is associated with second-order cones. For now we assume that all vectors consist of a single block $x=\left(x_{0} ; \bar{x}\right) \in R^{n}$. For two vectors $x$ and $y$, define the following multiplication:

$$
x \circ z=\left(x^{T} z ; x_{0} \bar{z}+z_{0} \bar{x}\right)=\operatorname{Arw}(x) z=\operatorname{Arw}(x) \operatorname{Arw}(z) e .
$$

So, “+", "o" with $e=(1 ; 0) \in R \times R^{n-1}$ give rise to a Jordan algebra associated with second-order cone $K$.

It is well known that the vector $x \in R^{n}$ has a spectral decomposition as

$$
x=\lambda_{1} c_{1}+\lambda_{2} c_{2}
$$

where $\lambda_{1}, \lambda_{2}$ and $c_{1}, c_{2}$ are the spectral values and spectral vectors of $x$ given by

$$
\begin{gathered}
\lambda_{i}=x_{0}+(-1)^{i}\|\bar{x}\|, \\
c_{i}= \begin{cases}\frac{1}{2}\left(1 ;(-1)^{i} \frac{\bar{x}}{\|\bar{x}\|}\right), & \text { if } \bar{x} \neq 0, \\
\frac{1}{2}\left(1 ;(-1)^{i} \omega\right), & \text { if } \bar{x}=0,\end{cases}
\end{gathered}
$$

where $i=1,2$, and $\omega$ is any vector in $R^{n-1}$ satisfying $\|\omega\|=1$.

The KKT optimality condition of problem (1) is written as follows:

$$
\begin{gathered}
A x-b=0, \\
A^{T} y+z-c=0, \\
x, z \in K, \quad x \circ z=0 .
\end{gathered}
$$

Interior point methods typically deal with the following perturbation of the optimality condition (8):

$$
\begin{gathered}
A x-b=0, \\
A^{T} y+z-c=0, \\
x, z \in K, \quad x \circ z=\mu^{2} e .
\end{gathered}
$$

In this paper, we structure a nonlinear system that is equivalent to (9). Then the nonlinear system is solved by quasi-Newton method to get the optimum solution of (1). Here, we introduce a smoothing function $\varphi(x, z, \mu): R^{n} \times$ $R^{n} \times R \rightarrow R^{n}$

$$
\varphi(x, z, \mu)=x+z-\sqrt{x^{2}+z^{2}+2 \mu^{2} e} .
$$

Reference [5] gives some properties of smoothing function (10).
Proposition 1. $\varphi(x, z, 0)=0$ if and only if $x \succcurlyeq_{K} 0, z \geqslant_{K} 0$ and $x \circ z=0$.

Proposition 2. For any $\mu>0, \varphi(x, z, \mu)=0$ if and only if $x>_{K} 0, z>_{K} 0$ and $x \circ z=\mu^{2} e$.

$$
\text { Let } \begin{aligned}
F: R^{n} \times R^{m} \times R^{n} \times R \rightarrow R^{m} \times R^{n} \times R^{n} \times R, \\
F(x, y, z, \mu)=\left(\begin{array}{c}
A x-b \\
A^{T} y+z-c \\
\varphi(x, z, \mu) \\
\mu
\end{array}\right) .
\end{aligned}
$$

Apparently, $F(x, y, z, \mu)=0$ is equivalent to (8). Let $(x, y, z, \mu)=u$, then $F(x, y, z, \mu)=F(u)$. So, the KKT optimality condition is equivalent to the following:

$$
F(u)=0 .
$$

Next, we solve (12) by using Broyden rank one quasiNewton method. When we solve the problem (12) with quasiNewton method, the gradient or Jacobian does not appear. It can reduce the amount of calculation. While it is not suitable to use the usual line search such as Wolf or Powll rules. Thus, we suggest two kinds of derivative-free line search rule.

In 1986, Griewank [6] put forward a kind of monotonous line search. Set

$$
q_{k}(\alpha)=-\frac{F\left(u_{k}\right)^{T}\left(F\left(u_{k}+\alpha d_{k}\right)-F\left(u_{k}\right)\right)}{\left\|F\left(u_{k}+\alpha d_{k}\right)-F\left(u_{k}\right)\right\|^{2}} .
$$

Let $\alpha_{k}$ satisfy the following inequality:

$$
q_{k}(\alpha) \geq \frac{1}{2}+\varepsilon
$$

where $\varepsilon \in(0,1 / 6)$ is a constant.

Due to (13) and (14), we obtain

$$
\begin{aligned}
& \left\|F\left(u_{k}+\alpha d_{k}\right)\right\|^{2} \\
& \quad \leq\left\|F\left(u_{k}\right)\right\|^{2}-2 \varepsilon\left\|F\left(u_{k}+\alpha d_{k}\right)-F\left(u_{k}\right)\right\|^{2} \\
& \quad \leq\left\|F\left(u_{k}\right)\right\|^{2} .
\end{aligned}
$$

Clearly, the line search is a normal descent method.

From the definition of $q_{k}(\alpha)$, the following conclusion holds. When $F\left(u_{k}\right)^{T} F^{\prime}\left(u_{k}\right) d_{k} \neq 0$, (14) holds for all $\alpha_{k}$ sufficiently small. When $F\left(u_{k}\right)^{T} F^{\prime}\left(u_{k}\right) d_{k}=0$, (14) does not hold for any $\alpha_{k}$.

Because there is a failure in the line search rule, many scholars put forward some kinds of different derivative-free line search rules. In [7], Li and Fukushima suggested a kind of nonmonotone derivative-free line search rules. The step $\alpha_{k}$ satisfies the following inequality:

$$
\begin{aligned}
& \left\|F\left(u_{k}+\alpha_{k} d_{k}\right)\right\|^{2} \\
& \quad \leq\left\|F\left(u_{k}\right)\right\|^{2}-\sigma_{1}\left\|\alpha_{k} F\left(u_{k}\right)\right\|^{2}-\sigma_{2}\left\|\alpha_{k} d_{k}\right\|^{2}+\eta_{k}\left\|F\left(u_{k}\right)\right\|^{2},
\end{aligned}
$$


where, $\sigma_{1}, \sigma_{2}>0$ are constants, and there exists a constant $\eta>0$, such that the positive sequence $\left\{\eta_{k}\right\}$ satisfies

$$
\sum_{k=0}^{\infty} \eta_{k} \leq \eta<\infty
$$

Obviously, (16) holds for all $\alpha_{k}>0$ sufficiently small. For any $k$, we have

$$
\left\|F\left(u_{k}+\alpha_{k} d_{k}\right)\right\|^{2} \leq\left(1+\eta_{k}\right)\left\|F\left(u_{k}\right)\right\|^{2} .
$$

According to the introduction, we know that there is a defect in the line search rule (15), but it is monotonically decreasing, namely, $\left\|F\left(u_{k+1}\right)\right\| \leq\left\|F\left(u_{k}\right)\right\|$. However (16) is non-monotone line search rule. In this paper, the reasonable combination of the two line search rules is given.

In order to implement the mixed line search rules, we define the function $f: R \rightarrow R$

$$
f(\alpha)=\frac{\left\|F\left(u_{k}+\alpha d_{k}\right)\right\|^{2}-\left\|F\left(u_{k}\right)\right\|^{2}}{\alpha} .
$$

Apparently, $\lim _{\alpha \rightarrow 0} f(\alpha)=F\left(u_{k}\right)^{T} F^{\prime}\left(u_{k}\right) d_{k}$. When $\alpha$ and $|f(\alpha)|$ are too small, (14) does not hold. We will use the nonmonotone line search.

On the basis of the previous section, we will suggest a Broyden rank one quasi-Newton method for solving SOCP in this section. Now we present an algorithm for solving (12).

\section{Algorithm A.}

Step 0. Initialization and Date. Choose parameters $\lambda, \delta \in$ $(0,1), \sigma_{1}, \sigma_{2}, \sigma_{3}>0, \varepsilon, \varepsilon_{0}>0$. Fix a positive sequence $\left\{\eta_{k}\right\}$ that satisfies (17). Fix a starting point $u_{0} \in R^{2 n+m+1}$, and an initial matrix $B_{0} \in R^{(2 n+m+1) \times(2 n+m+1)}$. Set $k=0$.

Step 1. Termination Conditions. If $F\left(u_{k}\right)=0$, stop; otherwise, let $d_{k}$ be a solution of the linear equation

$$
B_{k} d_{k}+F\left(u_{k}\right)=0 .
$$

Step 2. The Line Search for Unit Step. If

$$
\left\|F\left(u_{k}+d_{k}\right)\right\| \leq \lambda\left\|F\left(u_{k}\right)\right\|-\sigma_{3}\left\|d_{k}\right\|^{2}
$$

holds, then set $\alpha_{k}=1$. Go to Step 4 .

Step 3. Mixed Line Search Rule.

(3.1) Set $i=0$;

(3.2) If $\delta^{i}$ satisfies (14), set $\alpha_{k}=\delta^{i}$; otherwise, if $\left|f\left(\delta^{i}\right)\right| \leq$ $\varepsilon_{0}$ holds, let $\alpha_{k}$ be the maximum of the numbers $\left\{1, \delta^{1}, \delta^{2}, \ldots\right\}$ satisfying (16). Else, set $i=i+1$. Go back to Step (3.2).

Step 4. Update. Set $u_{k+1}=u_{k}+\alpha_{k} d_{k}$.

Step 5. Computation of $B_{k+1}$. By the Broyden rank one correction formula, we obtain

$$
B_{k+1}=B_{k}+\frac{\left(y_{k}-B_{k} s_{k}\right) s_{k}^{T}}{\left\|s_{k}\right\|^{2}},
$$

where $s_{k}=u_{k+1}-u_{k}, y_{k}=F\left(u_{k+1}\right)-F\left(u_{k}\right)$, and $B_{k+1}$ is nonsingular.

Step 6. Set $k=k+1$. Go back to Step 1 .

\section{Global Convergence}

In this section, we prove the global convergence of algorithm. For this reason, we define some variables in the algorithm and give some hypotheses.

We define

$$
G_{k+1}=\int_{0}^{1} F^{\prime}\left(u_{k}+\tau s_{k}\right) d \tau,
$$

then $y_{k}=G_{k+1} s_{k}$. Set

$$
\xi_{k}=\frac{\left\|y_{k}-B_{k} s_{k}\right\|}{\left\|s_{k}\right\|}
$$

then

$$
\xi_{k}=\frac{\left\|\left(G_{k+1}-B_{k}\right) s_{k}\right\|}{\left\|s_{k}\right\|}=\frac{\left\|\left(G_{k+1}-B_{k}\right) d_{k}\right\|}{\left\|d_{k}\right\|} .
$$

Proposition 3. The sequence $\left\{u_{k}\right\}$ which is generated by the algorithm satisfies

$$
\left\|F\left(u_{k}\right)\right\| \leq\left\|F\left(u_{k-1}\right)\right\|
$$

then the level set

$$
\Omega=\left\{u \in R^{2 n+m+1} \mid\left\|F\left(u_{k+1}\right)\right\|^{2} \leq e^{\eta}\left\|F\left(u_{0}\right)\right\|^{2}\right\}
$$

is bounded, where $\eta$ is the constant defined in (17).

Proposition 4. $F^{\prime}(u)$ is Lipschitz continuous on $R^{2 n+m+1}$; that is, there exists a constant $L>0$ such that

$$
\left\|F^{\prime}(u)-F^{\prime}(v)\right\| \leq L\|u-v\|, \quad \forall u, v \in R^{2 n+m+1} .
$$

Proof. By [5], $\nabla \varphi(x, z, \mu)$ is continuously differentiable function, then there exists a constant $L>0$ such that

$$
\begin{aligned}
& \left\|\nabla \varphi\left(x_{1}, z_{1}, \mu_{1}\right)-\nabla \varphi\left(x_{2}, z_{2}, \mu_{2}\right)\right\| \\
& \quad \leq L\left\|\left(x_{1}, z_{1}, \mu_{1}\right)-\left(x_{2}, z_{2}, \mu_{2}\right)\right\|, \quad \forall x_{1}, x_{2}, z_{1}, z_{2}, \mu_{1}, \mu_{2} .
\end{aligned}
$$

For any $u, v \in R^{2 n+m+1}$, we have

$$
\begin{aligned}
\left\|F^{\prime}(u)-F^{\prime}(v)\right\| & =\left\|\nabla \varphi\left(x_{u}, z_{u}, \mu_{u}\right)-\nabla \varphi\left(x_{v}, z_{v}, \mu_{v}\right)\right\| \\
& \leq L\left\|\left(x_{u}, z_{u}, \mu_{u}\right)-\left(x_{v}, z_{v}, \mu_{v}\right)\right\| \\
& \leq L\|u-v\| .
\end{aligned}
$$

(H1) The matrix $A$ has full of row rank. 
From Proposition 3, Proposition 4, and (H1), $F^{\prime}(u)$ is reversible for all $u \in \Omega$. There exist constants $t^{\prime}, 0<t \leq T$, $0<T_{1} \leq T_{2}$, and $0<\tilde{t} \leq T_{3}$, such that

$$
\begin{gathered}
t^{\prime}\|u-v\| \leq\|F(u)-F(v)\| \leq T^{\prime}\|u-v\|, \\
\tilde{t}\left\|s_{k}\right\| \leq\left\|F^{\prime}\left(u_{k}\right) s_{k}\right\| \leq T_{3}\left\|s_{k}\right\|, \\
T_{1} \leq\left\|\left(F^{\prime}(u)\right)^{-1}\right\| \leq T_{2}, \\
t \leq\left\|F^{\prime}(u)\right\| \leq T .
\end{gathered}
$$

Lemma 5. If the sequence $\left\{u_{k}\right\}$ is generated by the algorithm, then $\left\{u_{k}\right\} \subset \Omega$.

Proof. If $\alpha_{k}$ is defined by Step 2 or (14), then we have

$$
\left\|F\left(u_{k+1}\right)\right\| \leq\left\|F\left(u_{k}\right)\right\| .
$$

The above inequality and (18) imply

$$
\left\|F\left(u_{k+1}\right)\right\|^{2} \leq\left(1+\eta_{k}\right)\left\|F\left(u_{k}\right)\right\|^{2},
$$

where the sequence $\left\{u_{k}\right\}$ is generated by the algorithm. According to (36), we have

$$
\begin{aligned}
\| F & \left(u_{k+1}\right) \|^{2} \\
& \leq\left(1+\eta_{k}\right)\left\|F\left(u_{k}\right)\right\|^{2} \leq \cdots \leq\left\|F\left(u_{0}\right)\right\|^{2} \prod_{j=0}^{k}\left(1+\eta_{j}\right) \\
& \leq\left\|F\left(u_{0}\right)\right\|^{2}\left(\frac{1}{1+k} \sum_{j=0}^{k}\left(1+\eta_{j}\right)\right)^{k+1} \\
& =\left\|F\left(u_{0}\right)\right\|^{2}\left(1+\frac{1}{1+k} \sum_{j=0}^{k} \eta_{j}\right)^{k+1} \\
& \leq\left\|F\left(u_{0}\right)\right\|^{2}\left(1+\frac{\eta}{1+k}\right)^{k+1} \leq e^{\eta}\left\|F\left(u_{0}\right)\right\|^{2}
\end{aligned}
$$

Hence, $\left\{u_{k}\right\} \subset \Omega$. The claim holds.

Lemma 6. If (H1) holds and the sequence $\left\{u_{k}\right\}$ is generated by the algorithm, then

$$
\sum_{k=0}^{\infty}\left\|s_{k}\right\|^{2}<\infty
$$

Proof. If $\alpha_{k}$ is determined by (16), it is clearly known that

$$
\sigma_{2}\left\|s_{k}\right\|^{2} \leq\left\|F\left(u_{k}\right)\right\|^{2}-\left\|F\left(u_{k}+\alpha_{k} d_{k}\right)\right\|^{2}+\eta_{k}\left\|F\left(u_{k}\right)\right\|^{2} .
$$

If $\alpha_{k}$ is determined by Step 2 , from it can be known that

$$
\sigma_{3}\left\|s_{k}\right\|^{2} \leq\left\|F\left(u_{k}\right)\right\|-\left\|F\left(u_{k+1}\right)\right\|+\eta_{k}\left\|F\left(u_{k}\right)\right\|^{2} .
$$

If $\alpha_{k}$ is ascertained by (14), then from (32), we have

$$
\begin{aligned}
& \left\|F\left(u_{k}+\alpha_{k} d_{k}\right)\right\|^{2} \\
& \quad \leq\left\|F\left(u_{k}\right)\right\|^{2}-2 \varepsilon\left\|F\left(u_{k}+\alpha_{k} d_{k}\right)-F\left(u_{k}\right)\right\|^{2} \\
& \quad \leq\left\|F\left(u_{k}\right)\right\|^{2}-2 \varepsilon t^{\prime 2}\left\|s_{k}\right\|^{2} .
\end{aligned}
$$

Thus we obtain

$$
2 \varepsilon t^{\prime 2}\left\|s_{k}\right\|^{2} \leq\left\|F\left(u_{k}\right)\right\|^{2}-\left\|F\left(u_{k+1}\right)\right\|^{2}+\eta_{k}\left\|F\left(u_{k}\right)\right\|^{2} .
$$

Set $\sigma_{0}=\min \left\{\sigma_{2}, 2 \varepsilon t^{\prime 2}\right\}$. Then, from (39) and (42), we see that

$$
\begin{aligned}
\sigma_{0}\left\|s_{k}\right\|^{2} \leq & \left\|F\left(u_{k}\right)\right\|^{2}-\left\|F\left(u_{k+1}\right)\right\|^{2}+\eta_{k}\left\|F\left(u_{k}\right)\right\|^{2} \\
= & \left(\left\|F\left(u_{k}\right)\right\|-\left\|F\left(u_{k+1}\right)\right\|\right) \\
& \times\left(\left\|F\left(u_{k}\right)\right\|+\left\|F\left(u_{k+1}\right)\right\|\right)+\eta_{k}\left\|F\left(u_{k}\right)\right\|^{2} \\
\leq & 2 \sqrt{e^{\eta}}\left\|F\left(u_{0}\right)\right\| \\
& \times\left(\left\|F\left(u_{k}\right)\right\|-\left\|F\left(u_{k+1}\right)\right\|\right)+\eta_{k}\left\|F\left(u_{k}\right)\right\|^{2} .
\end{aligned}
$$

Let $\bar{\sigma}=\min \left\{\sigma_{0}, \sigma_{3}\right\}$ and $C=\max \left\{1,2 \sqrt{e^{\eta}}\left\|F\left(u_{0}\right)\right\|\right\}$. It follows directly from (40) and (43) that

$$
\bar{\sigma}\left\|s_{k}\right\|^{2} \leq C\left(\left\|F\left(u_{k}\right)\right\|-\left\|F\left(u_{k+1}\right)\right\|\right)+\eta_{k}\left\|F\left(u_{k}\right)\right\|^{2} .
$$

By summing both sides of the above inequality, we have

$$
\begin{aligned}
\bar{\sigma} \sum_{k=0}^{\infty}\left\|s_{k}\right\|^{2} & \leq C\left\|F\left(u_{0}\right)\right\|+\sum_{k=0}^{\infty} \eta_{k}\left\|F\left(u_{k+1}\right)\right\|^{2} \\
& \leq C\left\|F\left(u_{0}\right)\right\|+e^{\eta}\left\|F\left(u_{0}\right)\right\|^{2} \sum_{k=0}^{\infty} \eta_{k} \\
& \leq C\left\|F\left(u_{0}\right)\right\|+e^{\eta} \eta\left\|F\left(u_{0}\right)\right\|^{2} .
\end{aligned}
$$

This completes the proof.

According to some results in $[8,9]$, we can obtain the following results.

Lemma 7. Let the positive sequence $\left\{a_{k}\right\}$ and $\left\{t_{k}\right\}$ satisfy $a_{k+1} \leq$ $\left(1+t_{k}\right) a_{k}+t_{k}$ and $\sum_{k=0}^{\infty} t_{k}<\infty$, then the sequence $\left\{a_{k}\right\}$ converges.

Lemma 8. Let the sequence $\left\{u_{k}\right\}$ be generated by the algorithm, then the sequence $\left\{\left\|F\left(u_{k}\right)\right\|^{2}\right\}$ is convergent.

Lemma 9. Let (H1) hold. Let the sequence $\left\{\xi_{k}\right\}$ be defined by (24) and let $\left\{u_{k}\right\}$ be generated by the algorithm. If

$$
\sum_{k=0}^{\infty}\left\|s_{k}\right\|^{2}<\infty,
$$

then

$$
\lim _{k \rightarrow \infty} \frac{1}{k} \sum_{j=0}^{k-1} \xi_{j}^{2}=0 .
$$

Specifically, there is a subsequence of $\left\{\xi_{k}\right\}$ which converges to zero. Moreover, if

$$
\sum_{k=0}^{\infty}\left\|s_{k}\right\|<\infty,
$$


then

$$
\sum_{k=0}^{\infty} \xi_{k}^{2}<\infty
$$

In particular, the whole sequence $\left\{\xi_{k}\right\} \rightarrow 0$.

Lemma 10. Let (H1) hold and the sequence $\left\{u_{k}\right\},\left\{d_{k}\right\}$ be generated by the algorithm. There exist sthe subsequence $\left\{u_{k}, d_{k}\right\}_{k \in B}$ of $\left\{u_{k}, d_{k}\right\}$ that converges to $\{\bar{u}, \bar{d}\}$, respectively. Furthermore, one has

$$
F^{\prime}(\bar{u}) \bar{d}+F(\bar{u})=0 .
$$

Proof. Let $G_{k+1}$ and $\xi_{k}$ be defined by (23) and (24), respectively. By Lemmas 6 and 9, there is a subsequence of $\left\{\xi_{k}\right\}$ tending to zero, from which Lemma 5 implies that $\left\{u_{k}\right\} \subset \Omega$ is bounded. Without loss of generality, we assume that there is a subsequence $\left\{u_{k}\right\}$ that converges to $\bar{u}$. Since Lemma 6 implies $s_{k}=u_{k+1}-u_{k} \rightarrow 0$, then $G_{k+1}=\int_{0}^{1} F^{\prime}\left(u_{k}+\tau s_{k}\right) d \tau \rightarrow F^{\prime}(\bar{u})$, $(k \rightarrow \infty)$. Therefore, there exists a constant $T_{4}>0$ such that $\left\|G_{k+1}^{-1}\right\| \leq T_{4}$ for all $k \in N$ sufficiently large. Thus from (20) and (25), we obtain

$$
\begin{aligned}
\left\|d_{k}\right\| & =\left\|d_{k}-G_{k+1}^{-1}\left(B_{k} d_{k}+F\left(u_{k}\right)\right)\right\| \\
& =\left\|G_{k+1}^{-1}\left(\left(G_{k+1}-B_{k}\right) d_{k}-F\left(u_{k}\right)\right)\right\| \\
& \leq\left\|G_{k+1}^{-1}\right\|\left(\left\|\left(G_{k+1}-B_{k}\right) d_{k}\right\|+\left\|F\left(u_{k}\right)\right\|\right) \\
& \leq T_{4}\left(\xi_{k}\left\|d_{k}\right\|+\left\|F\left(u_{k}\right)\right\|\right) .
\end{aligned}
$$

So there is a constant $E$ such that, for all $k \in B$ sufficiently large,

$$
\left\|d_{k}\right\| \leq E\left\|F\left(u_{k}\right)\right\| .
$$

Without loss of generality, we assume that $\left\{d_{k}\right\}_{k \in B}$ converges to $\bar{d}$ (necessarily, we can treat the sequence $B$ as its some subsequence). By (25), we obtain $\left\|B_{k} d_{k}-G_{k+1} d_{k}\right\|=\xi_{k}\left\|d_{k}\right\|$. It follows that $B_{k} d_{k} \rightarrow F^{\prime}(\bar{u}) \bar{d}$ as $k \rightarrow \infty$ with $k \in B$. Thus, taking the limit in (20) as $k \rightarrow \infty$ with $k \in B$, yields (50). The proof is finished.

Theorem 11. Assume that (H1) holds, then the whole sequence $\left\{u_{k}\right\}$ generated by the algorithm converges to the unique solution of (12).

Proof. It is clear from Lemma 8 that the sequence $\left\{\left\|F\left(u_{k}\right)\right\|^{2}\right\}$ is convergent. Thus, it is sufficient to verify that there exists an accumulation point of $\left\{u_{k}\right\}$ that is the solution of (12).

(i) We suppose that there are an infinite number of $k$ such that $\alpha_{k} s$ are determined by (21). Let $B=\left\{j_{1}, j_{2}, \ldots\right\}$ be an index set with $B=\left\{k \mid \alpha_{k}\right.$ being determined by (21) $\}$. If $k \in B$, then $\left\|F\left(u_{k+1}\right)\right\| \leq \lambda\left\|F\left(u_{k}\right)\right\|$ holds.

If $k \notin B$, then we have $\left\|F\left(u_{k+1}\right)\right\|^{2} \leq\left(1+\eta_{k}\right)\left\|F\left(u_{k}\right)\right\|^{2}$, in that way, $\left\|F\left(u_{k+1}\right)\right\| \leq\left(1+\eta_{k}\right)\left\|F\left(u_{k}\right)\right\|$. This implies that $\left\|F\left(u_{j_{k}+1}\right)\right\| \leq \lambda^{k} e^{\eta}\left\|F\left(u_{j_{0}}\right)\right\|$. Hence, $\lim _{k \in B}\left\|F\left(u_{j_{k}}\right)\right\|=0$.

(ii) If there exists an infinite number of $k$ such that $\alpha_{k} s$ are determined by (14). Let $B_{1}$ be an index set with
$B_{1}=\left\{k \mid \alpha_{k}\right.$ is determined by (14) $\}$. Since $\left\{\alpha_{k}\right\}$ is bounded, there is a subsequence $\left\{\alpha_{k}\right\}_{k \in B_{2} \subset B_{1}}$ of $\left\{\alpha_{k}\right\}_{k \in B_{1}}$ that tends to $\bar{\alpha} \geq 0$, that is, $\lim _{k \in B_{2}} \alpha_{k}=\bar{\alpha}$. If $\bar{\alpha} \neq 0$, then $\bar{\alpha} \bar{d}=0$ implies that $\bar{d}=0$. By (50), we obtain $F(\bar{u})=0$.

If $\bar{\alpha}=0$, then $\alpha_{k} \rightarrow 0^{+}$for $k \rightarrow \infty$ with $k \in B_{2}$. By Step 3 of the algorithm, $\widetilde{\alpha}_{k}=\alpha_{k} / \delta$ does not satisfy (14) for all $k \in B_{2}$ large enough, namely,

$$
\begin{aligned}
& -F\left(u_{k}\right)^{T}\left(F\left(u_{k}+\widetilde{\alpha}_{k} d_{k}\right)-F\left(u_{k}\right)\right) \\
& \quad \leq\left(\frac{1}{2}+\varepsilon\right)\left\|F\left(u_{k}+\widetilde{\alpha}_{k} d_{k}\right)-F\left(u_{k}\right)\right\|^{2} .
\end{aligned}
$$

Thus,

$$
\begin{aligned}
\lim _{k \in B_{2}} & -\frac{F\left(u_{k}\right)^{T}\left(F\left(u_{k}+\widetilde{\alpha}_{k} d_{k}\right)-F\left(u_{k}\right)\right)}{\widetilde{\alpha}_{k}} \\
& \leq \lim _{k \in B_{2}}\left(\frac{1}{2}+\varepsilon\right)\left\|\frac{F\left(u_{k}+\widetilde{\alpha}_{k} d_{k}\right)-F\left(u_{k}\right)}{\widetilde{\alpha}_{k}}\right\|^{2} \widetilde{\alpha}_{k},
\end{aligned}
$$

that is,

$$
F(\bar{u})^{T} F^{\prime}(\bar{u}) \bar{d} \geq 0 .
$$

By (50), we get $F(\bar{u})=0$.

(iii) According to (ii), without loss of generality, we suppose that all $\alpha_{k} s$ are determined by (16) for all $k$ sufficiently large. Let $B_{4}$ be an index set with $B_{4}=\{k \mid$ $\alpha_{k}$ is determined by (16)\}. Set

$$
\lim _{k \in B_{4}, k \rightarrow \infty} \alpha_{k}=\alpha^{\prime},
$$

then $\alpha^{\prime} \geq 0$ and $\alpha^{\prime} \bar{d}=0$. If $\alpha>0$, then $\bar{d}=0$. Thus from (50), we obtain $F(\bar{u})=0$. Otherwise, $\alpha^{\prime}=0$, that is,

$$
\lim _{k \in B_{4}, k \rightarrow \infty} \alpha_{k}=0 .
$$

By using Step 3 of the algorithm, we know that $\alpha^{\prime}=\alpha_{k} / \delta$ does not satisfy (16) for all $k \in B_{4}$ sufficiently large. Therefore, we have

$$
\begin{aligned}
& \left\|F\left(u_{k}+\alpha_{k}^{\prime} d_{k}\right)\right\|^{2}-\left\|F\left(u_{k}\right)\right\|^{2} \\
& >-\sigma_{1}\left\|\alpha_{k}^{\prime} F\left(u_{k}\right)\right\|^{2}-\sigma_{2}\left\|\alpha_{k}^{\prime} d_{k}\right\|^{2}+\eta_{k}\left\|F\left(u_{k}\right)\right\|^{2} \\
& >-\sigma_{1}\left\|\alpha_{k}^{\prime} F\left(u_{k}\right)\right\|^{2}-\sigma_{2}\left\|\alpha_{k}^{\prime} d_{k}\right\|^{2} .
\end{aligned}
$$

Dividing the both sides by $\alpha_{k}^{\prime}$ and then taking the limit as $k \rightarrow$ $\infty$ with $k \in B_{4}$, we get

$$
\begin{aligned}
& \lim _{k \in B_{4}, k \rightarrow \infty} \frac{\left\|F\left(u_{k}+\alpha_{k}^{\prime} d_{k}\right)\right\|^{2}-\left\|F\left(u_{k}\right)\right\|^{2}}{\alpha_{k}^{\prime}} \\
& \quad \geq \lim _{k \in B_{4}, k \rightarrow \infty}-\sigma_{1} \alpha_{k}^{\prime}\left\|F\left(u_{k}\right)\right\|^{2}-\sigma_{2} \alpha_{k}^{\prime}\left\|d_{k}\right\|^{2},
\end{aligned}
$$

that is,

$$
F(\bar{u})^{T} F^{\prime}(\bar{u}) \bar{d} \geq 0 .
$$

It is clear from (50) that $F(\bar{u})=0$. Therefore the result holds. 


\section{Local Superlinear Convergence}

In this section, we prove the local superlinear convergence of the algorithm.

Lemma 12. If (H1) holds and the sequence $\left\{u_{k}\right\}$ is generated by the algorithm, then there exist a constant $\xi>0$ and an index $\bar{k}$ such that

$$
\alpha_{k}=1
$$

whenever $k \geq \bar{k}$ and $\xi_{k} \leq \xi$. Furthermore, the relation

$$
\left\|F\left(u_{k}+d_{k}\right)\right\| \leq \lambda\left\|F\left(u_{k}\right)\right\|-\sigma_{3}\left\|d_{k}\right\|^{2}<\lambda\left\|F\left(u_{k}\right)\right\|
$$

holds for all $k \geq \bar{k}$ such that $\xi_{k} \leq \xi$.

Proof. By Step 2 of the algorithm, there exists a constant $\xi$ such that (62) holds whenever $\xi_{k} \leq \xi$ and for all $k$ sufficiently large. It follows from Theorem 11 that the sequence $\left\{u_{k}\right\}$ converges to the unique solution of problem (12), and there exists a constant $T_{4}$ such that $\left\|G_{k+1}^{-1}\right\| \leq T_{4}$ for all $k$ large enough. Then, from (52), we see that there exist constant $\xi^{\prime}>0$ and $\bar{E}>0$ such that

$$
\left\|d_{k}\right\| \leq \bar{E}\left\|F\left(u_{k}\right)\right\|
$$

whenever $\xi_{k} \leq \xi^{\prime}$ and for all $k$ sufficiently large. Due to (20), we obtain

$$
\begin{aligned}
G_{k+1} & \left(u_{k}+d_{k}-u^{*}\right) \\
= & G_{k+1}\left(u_{k}+d_{k}-u^{*}\right)-\left(B_{k} d_{k}+F\left(u_{k}\right)\right) \\
= & G_{k+1}\left(u_{k}-u^{*}\right)+\left(G_{k+1}-B_{k}\right) d_{k}-F\left(u_{k}\right) \\
= & \left(G_{k+1}-F^{\prime}\left(u^{*}\right)\right)\left(u_{k}-u^{*}\right) \\
& +\left(G_{k+1}-B_{k}\right) d_{k}-F\left(u_{k}\right) \\
& +F^{\prime}\left(u^{*}\right)+F^{\prime}\left(u^{*}\right)\left(u_{k}-u^{*}\right) .
\end{aligned}
$$

Hence, we have

$$
\begin{gathered}
\left\|u_{k}+d_{k}-u^{*}\right\| \\
\leq\left\|G_{k+1}^{-1}\right\|\left(\left\|G_{k+1}-F^{\prime}\left(u^{*}\right)\right\|\left\|u_{k}-u^{*}\right\|\right. \\
+\left\|\left(G_{k+1}-B_{k}\right) d_{k}\right\| \\
+\| F\left(u_{k}\right)-F^{\prime}\left(u^{*}\right)-F^{\prime}\left(u^{*}\right) \\
\left.+\left(u_{k}-u^{*}\right) \|\right) \\
\leq T_{4}\left(\xi_{k}\left\|d_{k}\right\|+o\left(\left\|u_{k}-u^{*}\right\|\right)\right) \\
\leq T_{4}\left(\xi_{k} \bar{E}\left\|F\left(u_{k}\right)\right\|+o\left(\left\|u_{k}-u^{*}\right\|\right)\right) \\
\leq T_{4}\left(\bar{E} T^{\prime} \xi_{k}\left\|u_{k}-u^{*}\right\|+o\left(\left\|u_{k}-u^{*}\right\|\right)\right),
\end{gathered}
$$

where the last inequality follows from (31). This implies that

$$
\begin{aligned}
\left\|F\left(u_{k}+d_{k}\right)\right\| & =\left\|F\left(u_{k}+d_{k}\right)-F\left(u^{*}\right)\right\| \\
& \leq T^{\prime}\left\|u_{k}+d_{k}-u^{*}\right\| \\
& \leq T^{\prime} T_{4}\left(\bar{E} T^{\prime} \xi_{k}\left\|u_{k}-u^{*}\right\|+o\left(\left\|u_{k}-u^{*}\right\|\right)\right) .
\end{aligned}
$$

Since $\left\{u_{k}\right\} \rightarrow u^{*}$ holds, from (33), there exists $\widetilde{t}>0$ such that

$$
\left\|F\left(u_{k}\right)\right\|=\left\|F\left(u_{k}\right)-F\left(u^{*}\right)\right\| \geq \tilde{t}\left\|u_{k}-u^{*}\right\|
$$

for all $k$ large enough. Then by (63), (65), and (66), we obtain

$$
\begin{aligned}
&\left\|F\left(u_{k}+d_{k}\right)\right\|-\lambda\left\|F\left(u_{k}\right)\right\|+\sigma_{3}\left\|d_{k}\right\|^{2} \\
& \leq T^{\prime} T_{4}\left(\bar{E} T^{\prime} \xi_{k}\left\|u_{k}-u^{*}\right\|+o\left(\left\|u_{k}-u^{*}\right\|\right)\right) \\
&-\lambda \tilde{t}\left\|u_{k}-u^{*}\right\|+\sigma_{3} \bar{E}^{2} T^{\prime 2}\left\|u_{k}-u^{*}\right\|^{2} \\
&=\sigma_{3} \bar{E}^{2} T^{\prime 2}\left\|u_{k}-u^{*}\right\|^{2}+T_{4} \bar{E} T^{\prime 2} \xi_{k}\left\|u_{k}-u^{*}\right\| \\
&-\lambda \tilde{t}\left\|u_{k}-u^{*}\right\|+o\left(\left\|u_{k}-u^{*}\right\|\right) \\
&=-\left(\lambda \tilde{t}-Q \xi_{k}\right)\left\|u_{k}-u^{*}\right\|+o\left(\left\|u_{k}-u^{*}\right\|\right),
\end{aligned}
$$

where $Q=T_{4} \bar{E} T^{\prime 2}$. Let $\xi=\min \left\{\xi^{\prime}, \lambda \widetilde{t} / 2 Q\right\}$, then we have

$$
\begin{gathered}
\left\|F\left(u_{k}+d_{k}\right)\right\|-\lambda\left\|F\left(u_{k}\right)\right\|+\sigma_{3}\left\|d_{k}\right\|^{2} \\
\leq-\frac{1}{2} \lambda \tilde{t}\left\|u_{k}-u^{*}\right\|+o\left(\left\|u_{k}-u^{*}\right\|\right),
\end{gathered}
$$

whenever $\xi_{k} \leq \xi$. Hence, when $u_{k}$ is sufficiently close to $u^{*}$ for all $k$ large enough, $\alpha_{k}=1$ can satisfy (62), which prove the conclusion.

Theorem 13. Assume that (H1) holds, then the sequence $\left\{u_{k}\right\}$ generated by the algorithm converges superlinearly to the unique solution of (12).

Proof. From (65), it is sufficient to verify that the sequence $\left\{\xi_{k}\right\} \rightarrow 0$. Let $\xi$ and $\bar{k}$ be determined by Lemma 12. By Lemmas 6 and 9, we have

$$
\lim _{k \rightarrow \infty} \frac{1}{k} \sum_{i=0}^{k-1} \xi_{i}^{2}=0
$$

Then there exists an index $\widetilde{k}$ such that

$$
\frac{1}{k} \sum_{i=0}^{k-1} \xi_{i}^{2} \leq \frac{1}{2} \xi^{2},
$$

whenever $k \geq \widetilde{k}$. This implies that

$$
\sum_{i=0}^{k-1} \xi_{i}^{2} \leq \frac{k}{2} \xi^{2} .
$$


TABLE 1

\begin{tabular}{|c|c|c|c|c|c|}
\hline Itr & Prec & Time (s) & $P_{\mathrm{val}}$ & $D_{\mathrm{val}}$ & Gap \\
\hline 30 & $9.5303 E-04$ & 0.2023 & - & - & - \\
\hline 32 & $7.6490 E-05$ & 0.2113 & - & - & - \\
\hline 35 & $7.3088 E-06$ & 0.2140 & - & - & - \\
\hline 37 & $4.2229 E-07$ & 0.2159 & - & - & - \\
\hline 38 & $3.6169 E-08$ & 0.2172 & 31.36476337 & 31.36476321 & $1.5364 E-07$ \\
\hline
\end{tabular}

The above inequality implies that for any $k \geq \widetilde{k}$, there are at least $k / 2$ many $i \leq k$ such that $\xi_{i}^{2} \leq \xi^{2}$, that is, $\xi_{i} \leq \xi$. Let $k^{\prime}=\max \{\bar{k}, \widetilde{k}\}$, then, by Lemma 12 , for any $k \geq 2 k^{\prime}$, there exist at least $[k / 2]-k^{\prime}$ many $i \leq k$ such that $\alpha_{i}=1$ and

$$
\left\|F\left(u_{i+1}\right)\right\|=\left\|F\left(u_{i}+d_{i}\right)\right\| \leq \lambda\left\|F\left(u_{i}\right)\right\| .
$$

Therefore, we have

$$
\left\|F\left(u_{i+1}\right)\right\|^{2} \leq \lambda^{2}\left\|F\left(u_{i}\right)\right\|^{2} .
$$

Let $\theta_{k}$ be the index set for which (74) holds, then $\left|\theta_{k}\right| \geq k / 2-$ $k^{\prime}-1$. When $i \notin \theta_{k}, \alpha_{k}$ is determined by Step 3 . Then by (15) and (18), we get

$$
\left\|F\left(u_{i+1}\right)\right\|^{2} \leq\left(1+\eta_{i}\right)\left\|F\left(u_{i}\right)\right\|^{2} .
$$

Let $i=\left\{k^{\prime}, k^{\prime}+1, \ldots, k\right\}$. From (74) and (18), we obtain

$$
\begin{aligned}
\left\|F\left(u_{i+1}\right)\right\|^{2} & \leq \lambda^{2\left|\theta_{k}\right|}\left\|F\left(u_{k^{\prime}}\right)\right\|^{2} \prod_{i=k^{\prime}}^{k}\left(1+\eta_{i}\right) \\
& \leq \lambda^{k-2 k^{\prime}} e^{\eta}\left\|F\left(u_{k^{\prime}}\right)\right\|^{2},
\end{aligned}
$$

that is,

$$
\left\|F\left(u_{k+1}\right)\right\| \leq \lambda^{(k / 2)-k^{\prime}} e^{\eta / 2}\left\|F\left(u_{k^{\prime}}\right)\right\| .
$$

Since $\lambda \in(0,1)$,

$$
\sum_{k=0}^{\infty}\left\|F\left(u_{k}\right)\right\|<\infty
$$

Hence,

$$
\sum\left\|u_{k}-u^{*}\right\| \leq \frac{1}{\tilde{t}} \sum\left\|F\left(u_{k}\right)-F\left(u^{*}\right)\right\| .
$$

Since $\left\|s_{k}\right\|=\left\|u_{k+1}-u_{k}\right\| \leq\left\|u_{k+1}-u^{*}\right\|+\left\|u_{k}-u^{*}\right\|$ implies that

$$
\sum\left\|s_{k}\right\|<\infty .
$$

Therefore, $\xi_{k} \rightarrow 0$ as $k \rightarrow \infty$. The proof is finished.

\section{Numerical Experiments}

In this section, we carry out a number of numerical experiments based on Algorithm A. The results show that
Algorithm A is effective. The numerical experiments are implemented on MATLAB 7.8.0.

The following parameter values were used:

$$
\begin{gathered}
\lambda=0.5, \quad \delta=0.5, \quad \sigma_{1}=0.5, \\
\sigma_{2}=0.5, \quad \sigma_{3}=0.5, \quad \varepsilon=0.1, \\
\varepsilon_{0}=0.01, \quad \eta_{k}=0.95^{k}, \quad \mu=1 .
\end{gathered}
$$

When the condition $\left\|F\left(u_{k}\right)\right\| \leq 10^{-7}$ holds, the algorithm stops.

In the tables of test results, itr denotes the number of iterations, prec the final residual of $\left\|F\left(u_{k}\right)\right\|$ when the algorithm stops, $P_{\text {val }}$ and $D_{\text {val }}$ the optimal values of the primal and dual problems of the test problems, and gap the dual gap of primal-dual problems. For the first nine experiments, the elements of the vectors $b, c$, matrix $A$, and initial points $x$, $y, z$ are random numbers from 0 to $10 . B_{0}$ is the identity matrix.

All of the following experiments are to solve the problem

$$
\begin{array}{lrrr}
\text { Primal } & \text { Dual } \\
\min & c^{T} x & \max & b^{T} y \\
\text { s.t. } & A x=b, & \text { s.t. } & A^{T} y+z=c, \\
& \quad x \succcurlyeq_{K} 0, & & z \succcurlyeq_{K} 0 .
\end{array}
$$

Example 14. The coefficients were chosen as

$$
A=\left[\begin{array}{llll}
4.0181 & 2.3992 & 1.8391 & 4.1727 \\
0.7597 & 1.2332 & 2.3995 & 0.4965
\end{array}\right],
$$

$$
\begin{gathered}
b^{T}=\left[\begin{array}{lll}
9.0272 & 9.4479
\end{array}\right], \\
c^{T}=\left[\begin{array}{llll}
7.8025 & 3.8974 & 2.4169 & 4.0391
\end{array}\right], \\
x^{T}=\left[\begin{array}{llll}
4.9086 & 4.8925 & 3.3772 & 9.0005
\end{array}\right], \\
y^{T}=\left[\begin{array}{lll}
3.6925 & 1.1120
\end{array}\right], \\
z^{T}=\left[\begin{array}{llll}
0.9645 & 1.3197 & 9.4205 & 9.5613
\end{array}\right],
\end{gathered}
$$

and the initial point was $(x, y, z, \mu)$. Let $r=1, K^{4} \mathrm{C}$ $R^{4}$. The data of the result of the problem is listed in Table 1 . 
TABle 2

\begin{tabular}{|c|c|c|c|c|c|}
\hline Itr & Prec & Time (s) & $P_{\mathrm{val}}$ & $D_{\text {val }}$ & Gap \\
\hline 35 & $8.3226 E-04$ & 0.2637 & - & - & - \\
\hline 37 & $7.8771 E-05$ & 0.2648 & - & - & - \\
\hline 40 & $5.6015 E-06$ & 0.2667 & - & - & - \\
\hline 42 & $1.9562 E-07$ & 0.2680 & - & - & - \\
\hline 44 & $2.9281 E-08$ & 0.2690 & 5.469380116 & 5.469380172 & $5.5606 E-08$ \\
\hline
\end{tabular}

TABLE 3

\begin{tabular}{|c|c|c|c|c|c|}
\hline Itr & Prec & Time (s) & $P_{\mathrm{val}}$ & $D_{\text {val }}$ & Gap \\
\hline 50 & $7.8376 E-04$ & 0.5057 & - & - & - \\
\hline 53 & $2.9878 E-05$ & 0.5100 & - & - & - \\
\hline 56 & $3.8683 E-06$ & 0.5130 & - & - & - \\
\hline 58 & $2.2355 E-07$ & 0.5157 & - & - & - \\
\hline 59 & $3.0909 E-08$ & 0.5167 & 0.605650674 & 0.605650683 & $9.4441 E-09$ \\
\hline
\end{tabular}

TABle 4

\begin{tabular}{lcccccccc}
\hline NO & $m$ & $n$ & Itr & Prec & Time $(\mathrm{s})$ & $P_{\text {val }}$ & $D_{\text {val }}$ \\
\hline exa4 & 4 & 8 & 57 & $4.0804 E-08$ & 0.3167 & -22.63388297 & -22.63388298 & $1.6941 E-08$ \\
exa5 & 5 & 10 & 59 & $6.9742 E-08$ & 0.1825 & 15.969930092 & 15.969930032 & $6.0249 E-08$ \\
exa6 & 6 & 10 & 61 & $5.4271 E-08$ & 0.2328 & 7.5547887021 & 7.5547887065 & $4.4554 E-09$ \\
exa7 & 6 & 12 & 70 & $8.3825 E-08$ & 0.2794 & 1.4491206670 & 1.4491206331 & $3.3890 E-08$ \\
exa8 & 7 & 14 & 77 & $6.2247 E-08$ & 0.2878 & 5.6139106732 & 5.6139106566 & $1.6642 E-08$ \\
exa9 & 8 & 16 & 110 & $3.8613 E-08$ & 0.3463 & 0.7337261831 & 0.7337262035 & $2.0411 E-08$ \\
\hline
\end{tabular}

Example 15. The coefficients were chosen as

$$
\begin{aligned}
& A= {\left[\begin{array}{llllll}
9.3194 & 0.5527 & 0.4500 & 1.1507 & 6.0609 & 9.9326 \\
0.9332 & 7.5716 & 8.4203 & 2.7156 & 6.7747 & 7.5754 \\
7.3878 & 4.6349 & 1.6471 & 3.1439 & 9.8764 & 2.7506
\end{array}\right], } \\
& b^{T}=\left[\begin{array}{llllll}
9.5401 & 4.1108 & 2.1662
\end{array}\right], \\
& c^{T}=\left[\begin{array}{llllll}
8.3755 & 7.4842 & 5.8386 & 1.6057 & 5.2884 & 4.6267
\end{array}\right], \\
& x^{T}=\left[\begin{array}{llllll}
6.2913 & 0.1487 & 0.4330 & 1.8026 & 2.0047 & 7.1936
\end{array}\right], \\
& y^{T}=\left[\begin{array}{lllll}
4.4287 & 8.4549 & 3.8979
\end{array}\right],
\end{aligned}
$$$$
z^{T}=\left[\begin{array}{llllll}
3.7954 & 0.9323 & 2.5915 & 3.3584 & 3.7511 & 1.4626
\end{array}\right],
$$

and the initial point was $(x, y, z, \mu)$. Let $r=2, K^{6}=K^{2} \times$ $K^{4}$. The data of the result of the problem is listed in the Table 2.
Example 16. The coefficients were chosen as

$$
\begin{aligned}
& A=\left[\begin{array}{llll}
4.2592 & 1.4806 & 8.2810 & 6.2869 \\
3.0049 & 9.5916 & 8.0789 & 1.1847 \\
8.8903 & 7.1447 & 9.0926 & 9.1897 \\
0.1737 & 3.0647 & 6.4281 & 6.2398
\end{array}\right. \\
& \begin{array}{llll}
2.5758 & 0.8805 & 5.6132 & 3.5499
\end{array} \\
& \begin{array}{lllll}
9.5130 & 5.1784 & 5.9816 & 2.4479
\end{array} \\
& \begin{array}{llll}
0.4621 & 8.7866 & 0.7505 & 1.0803
\end{array} \text {, } \\
& \begin{array}{llll}
0.2093 & 4.0218 & 3.9149 & 1.1229
\end{array} \\
& b^{T}=\left[\begin{array}{llll}
2.6720 & 2.6639 & 9.3608 & 1.8618
\end{array}\right] \\
& c^{T}=\left[\begin{array}{llll}
8.4678 & 6.5966 & 7.7966 & 3.5856
\end{array}\right. \\
& \left.\begin{array}{llll}
1.8414 & 0.1018 & 0.8591 & 3.2947
\end{array}\right], \\
& x^{T}=\left[\begin{array}{llll}
5.0744 & 1.4759 & 9.2070 & 9.2946
\end{array}\right. \\
& \left.\begin{array}{llll}
1.3675 & 8.7157 & 0.1236 & 7.2204
\end{array}\right], \\
& y^{T}=\left[\begin{array}{llll}
8.7859 & 3.8745 & 2.4643 & 1.1172
\end{array}\right] \\
& z^{T}=\left[\begin{array}{llll}
3.0083 & 5.0657 & 3.8661 & 9.3730
\end{array}\right. \\
& \left.\begin{array}{llll}
1.5954 & 7.7971 & 1.7746 & 8.0529
\end{array}\right]
\end{aligned}
$$


TABLE 5

\begin{tabular}{llccccccc}
\hline NO & $m$ & $n$ & Itr & Prec & Time $(s)$ & $P_{\text {val }}$ & $D_{\text {val }}$ \\
\hline exa10 & 10 & 20 & 94 & $8.8778 E-08$ & 0.6551 & 31.514993173 & 31.514993151 & $2.2923 E-08$ \\
exa11 & 15 & 28 & 117 & $5.4030 E-08$ & 0.9529 & 48.646522308 & 48.646522494 & $1.8531 E-07$ \\
exa12 & 20 & 40 & 198 & $8.3512 E-08$ & 2.3777 & 107.25705492 & 107.25705487 & $5.3633 E-10$ \\
\hline
\end{tabular}

and the initial point was $(x, y, z, \mu)$. Let $r=3, K^{8}=K^{2} \times$ $K^{3} \times K^{3}$. The data of the result of the problem is listed in the Table 3.

In Table 4, there are the final numerical results of the next six experiments, where the notations $m, n$ mean the number of the row and column of the matrix $A$, respectively, and NO denotes the number of the numerical experiments. In these experiments, we let $r=1$.

The ultimately numerical results of the last experiments were given in Table 5. All the matrices of $A$ that are in the examples are sparse matrices. The elements of these matrices $a_{i i}, a_{i, i+1}, a_{1, j}, a_{m, j}, i=1,2, \ldots, m, j=m+1, \ldots, n$, are random numbers from 0 to 10 . The rest elements of these matrices are zero. In exa10 and exa11, we let $r=1$. In exa12, we set $r=2$ and $K^{40}=K^{20} \times K^{20}$.

From Table 5, we can see Algorithm $A$ in this paper is efficient. The algorithm not only can solve the case of dense coefficient matrix $A$, but also can solve the case of sparse coefficient matrix $A$. From Table 5, we know that the cone $K$, whether or not partitioned the algorithm, is efficient.

\section{Acknowledgments}

This work was supported in part by NNSF (no. 11061011) of China and Guangxi Fund for Distinguished Young Scholars (2012GXSFFA060003) and Innovative project of Guangxi graduate education (2011105950701M26).

\section{References}

[1] Y. E. Nesterov and M. J. Todd, "Self-scaled barriers and interior-point methods for convex programming," Mathematics of Operations Research, vol. 22, no. 1, pp. 1-42, 1997.

[2] Y. E. Nesterov and M. J. Todd, "Primal-dual interior-point methods for self-scaled cones," SIAM Journal on Optimization, vol. 8, no. 2, pp. 324-364, 1998.

[3] X. N. Chi and S. Y. Liu, "A predictor-corrector smoothing method for second-order cone programming," Journal of Systems Science and Mathematical Sciences, vol. 29, no. 4, pp. 547$554,2009$.

[4] Y. J. Liu, L. W. Zhang, and Y. H. Wang, "Convergence properties of a smoothing method for linear second-order cone programming," Advances in Mathematics, vol. 36, no. 4, pp. 491-502, 2007.

[5] M. Fukushima, Z.-Q. Luo, and P. Tseng, "Smoothing functions for second-order-cone complementarity problems," SIAM Journal on Optimization, vol. 12, no. 2, pp. 436-460, 2001.

[6] A. Griewank, "The "global" convergence of Broyden-like methods with a suitable line search," The Journal of the Australian Mathematical Society B, vol. 28, no. 1, pp. 75-92, 1986.
[7] D. H. Li and M. Fukushima, "A derivative-free line search and DFP method for symmetric equations with global and superlinear convergence," Numerical Functional Analysis and Optimization, vol. 20, no. 1-2, pp. 59-77, 1999.

[8] J. E. Dennis Jr. and J. J. Moré, "A characterization of superlinear convergence and its application to quasi-Newton methods," Mathematics of Computation, vol. 28, pp. 549-560, 1974.

[9] D.-H. Li and M. Fukushima, "A derivative-free line search and global convergence of Broyden-like method for nonlinear equations," Optimization Methods and Software, vol. 13, no. 3, pp. 181-201, 2000. 


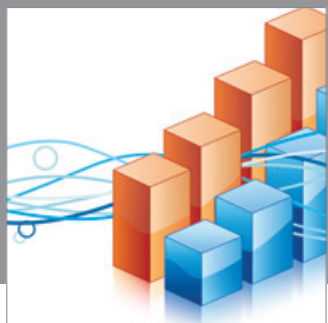

Advances in

Operations Research

mansans

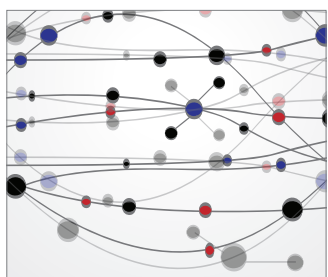

The Scientific World Journal
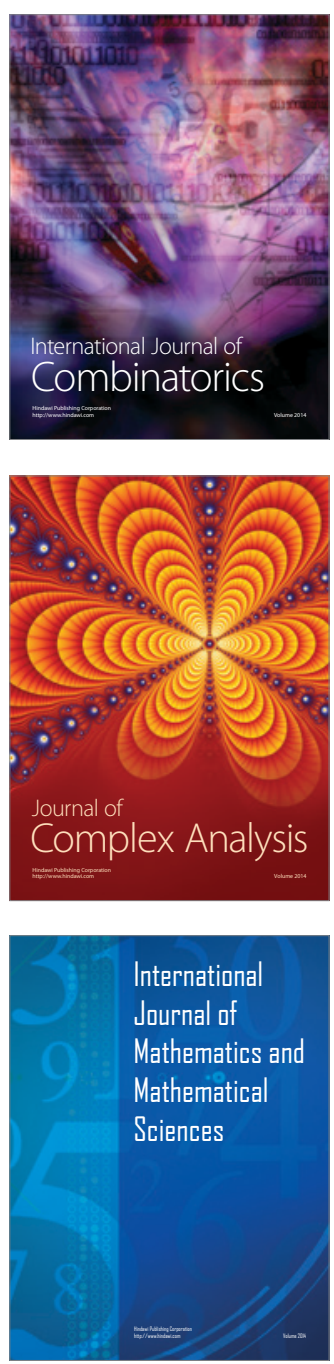
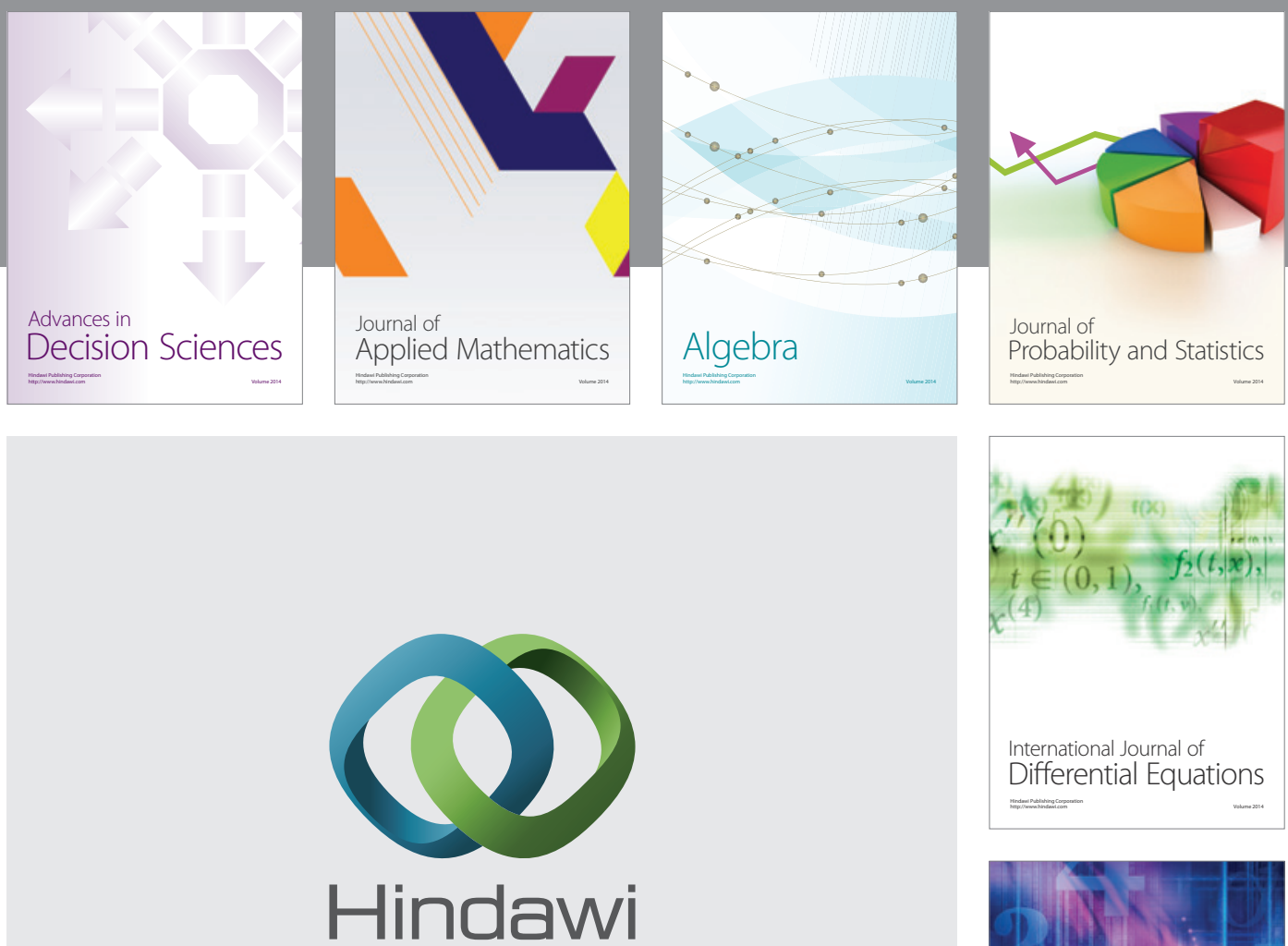

Submit your manuscripts at http://www.hindawi.com
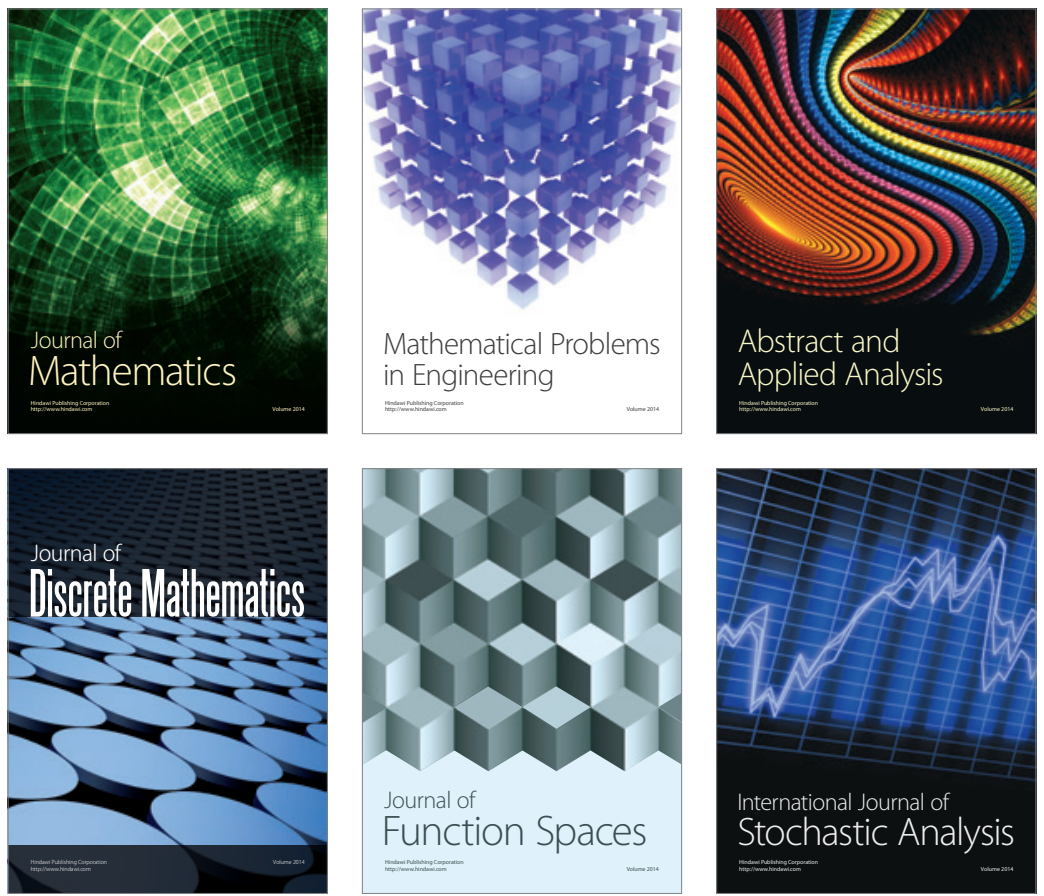

Journal of

Function Spaces

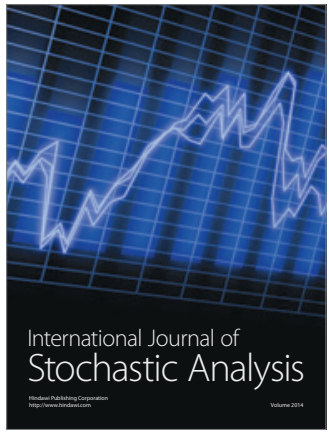

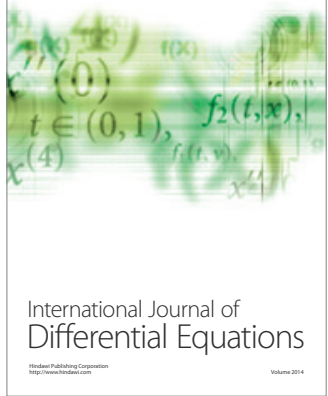
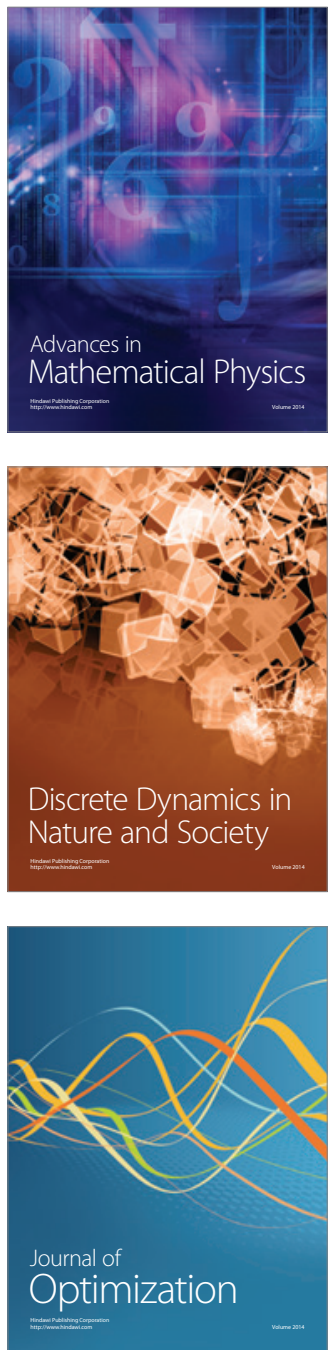